\title{
El uso de Internet como herramienta de socialización en adultos con parálisis cerebral en Brasil
}

\author{
The use of the Internet as a socialization tool in adults with cerebral \\ palsy in Brazil
}

\begin{abstract}
Resumen
La evidencia sobre la socialización a través del uso de Internet por parte de personas con discapacidad física, específicamente de adultos con parálisis cerebral, es aún escasa. En este artículo identificamos el potencial de esta tecnología como uno de los recursos para dicha finalidad. Fueron realizadas doce entrevistas semiestructuradas a adultos con parálisis cerebral del Estado de São Paulo, Brasil. Se sugieren cinco categorías temáticas de análisis que emergieron de las transcripciones de las entrevistas. La primera relacionada al uso de Internet para mantener y expandir sus relaciones interpersonales. La segunda relacionada a la utilidad de Internet como bienestar emocional para enfrentar situaciones críticas. La tercera relacionada con la visibilidad social de su condición, que ayudó a mostrar quiénes eran. La cuarta relacionada con su participación política, en la cual podían expresar sus puntos de vista y así hacer valer sus derechos legales. La última relacionada con las barreras actitudinales que las personas sin discapacidad asocian cuando este grupo poblacional hace uso de Internet. Se concluye que su uso tuvo principalmente un impacto positivo en la inclusión y participación de adultos con parálisis cerebral, mostrando una serie de posibilidades que promueven su acción social y política.
\end{abstract}

\section{Palabras clave}

Educación inclusiva, socialización, tecnologías digitales, Internet, adultos, discapacidad física, parálisis cerebral.

\begin{abstract}
The research studies of use of the Internet for socialization by people with physical disabilities such as adults with cerebral palsy are insufficient. In this paper, the author identifies the potential of this technology as a tool for socialization. Twelve adults with cerebral palsy were asked questions by using semi-structured interviews in the State of Sao Paulo, Brazil. Five thematic categories of analysis were suggested from the analysis and the interview transcripts. First category was related to the use of the Internet to maintain and expand interpersonal relationships. Second category was associated to the benefits of the Internet as an emotional well-being tool to fight critical situations. Third category was related to social visibility of their condition that helps to show who they are. Fourth category was related to the political participation where they can express their points of views and can have their legal rights. The final category was related to the attitudinal barriers that people without disabilities associate when this population group uses the Internet. In conclusion, the use of the Internet has a positive impact in inclusion and participation of adults with cerebral palsy by expanding their possibilities to promote their social and political participation.
\end{abstract}

\section{Keywords}

Inclusive education, socialization, digital technologies, Internet, adults, physical disability, cerebral palsy.

\author{
Iván Carlos Curioso-Vílchez \\ <icuriosov@gmail.com>
}

Universidade Estadual Paulista. Brasil

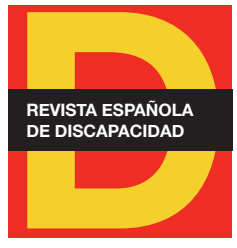

Para citar:

Curioso-Vílchez, I. C. (2021). El uso de Internet como herramienta de socialización en adultos con parálisis cerebral en Brasil. Revista Española de Discapacidad, 9(2), pp. 65-79.

Doi: <https://doi.org/10.5569/23405104.09.02.04>

Fecha de recepción: 22-03-2021 Fecha de aceptación: 25-10-2021 


\section{Introducción}

En la actualidad, los estudios sobre la socialización en los entornos virtuales como Internet es un tema emergente y de progresivo interés por diferentes especialidades. Sin embargo, existen vacíos de investigación sobre las relaciones y características del uso de Internet por adultos con discapacidad física como, por ejemplo, de aquellas y aquellos que poseen una parálisis cerebral (Caron y Light, 2015; Ferreira et al., 2012; González-Alonso y Matía, 2018; Leutar y Raič, 2008; Newman et al., 2017; Rasid y Nonis, 2015; Schur y Adya, 2013; Solís y Real, 2019; Suriá, 2012, 2017).

Las personas con esta discapacidad tienen dificultades en su coordinación motora, la cual afecta su desplazamiento para actividades cotidianas dentro de su hogar o fuera de éste, así como interfiere en su habilidad para la manipulación de determinados objetos. Además, sus músculos que producen el habla pueden estar comprometidos y, en otros casos, presentan una posible interferencia para la comunicación o comprensión con sus interlocutores (Panteliadis, 2018; Villalobos et al., 2016). A pesar de ello, este grupo de personas con parálisis cerebral disponen de las tecnologías digitales como el computador y los dispositivos móviles, entre otros recursos de comunicación alternativa o también denominadas tecnologías de apoyo o asistencia, para acceder a Internet (Braccialli et al., 2016; Heidrich y Bassani, 2012; Rodrigo y Tabuenca, 2020).

De acuerdo a una Investigación Nacional de Salud del año 2019, difundida por el Instituto Brasileño de Geografía y Estadística, existen 17,3 millones de personas en Brasil que tienen una discapacidad. De ese grupo, alrededor del $50 \%$ son personas que tienen 60 años o más (Cabral, 2021).

Respecto a la parálisis cerebral se señala la falta de estudios epidemiológicos actuales sobre esta condición en el Brasil (Peixoto et al., 2020). No obstante, se estima que la prevalencia a nivel mundial de la parálisis cerebral puede ser de 2,11 por cada 1000 nacidos vivos. Se resalta también la ausencia de investigaciones globales sobre la prevalencia de esta condición en adolescentes y adultos, al igual que sus experiencias de vida (Alves-Nogueira et al., 2020; Oskoui et al., 2013; Tonugble et al., 2021).

Particularmente, la literatura científica indica que, en el caso de los adultos con parálisis cerebral, se caracterizan por ser personas solteras, en aislamiento, con bajo índice de escolaridad y carencia de estudios superiores, así como se muestra una baja participación social en diferentes actividades de la vida diaria, entre otras manifestaciones, que destaca la falta de oportunidades para este grupo poblacional en la sociedad (GonzálezAlonso y Matía, 2018; Huskin et al., 2017; Smith et al., 2019; Solís y Real, 2019; Tonugble et al., 2021).

Contrariamente a dichas posturas, encontramos algunos estudios que revelan los procesos de socialización de las personas con parálisis cerebral en sus trayectorias de vida y además en los diferentes ámbitos presenciales como, por ejemplo, el educativo, laboral, recreativo y/o en espacios de terapia y rehabilitación (García et al., 2011; Jiménez, 2017; Maestro-González et al., 2018; Mendes et al., 2018; Santos et al., 2020; Sentenac et al., 2013). Si bien es cierto que en algunos de estos espacios se fortalecen y se amplían sus relaciones sociales, en otros, se promueven espacios que enfatizan situaciones de discriminación y exclusión por su misma condición de discapacidad (Huskin et al., 2017; Inahara, 2009; Mueller-Johnson et al., 2014; Ponsa et al., 2018; Wiegerink et al., 2006; Worrell, 2018).

En el contexto brasileño, los estudios sobre tecnologías como, por el ejemplo, el uso de la computadora e Internet por personas con parálisis cerebral nos muestra la carencia de investigaciones en adultos, a diferencia de niños y adolescentes (Braccialli et al., 2016; Heidrich et al., 2014). Específicamente, en el contexto 
internacional, para la población adulta con esta condición se evidencian escasos trabajos que indaguen sobre el impacto de Internet como una de las herramientas que posibilita su participación y afianzamiento para las relaciones sociales e interacciones entre sus pares (Caron y Light, 2015; Ferreira et al., 2012; Newman et al., 2017; Rasid y Nonis, 2015; Shah et al., 2020). A ello, se suma el paulatino incremento y uso de las redes sociales para personas con discapacidad física, que puede suplir la falta de accesibilidad arquitectónica para asistir a determinados lugares o ambientes de la ciudad, ya sea para encontrarse con familiares, amistades o para otros fines (González-Alonso y García-Alonso, 2014; Suriá, 2012, 2017).

Por lo mencionado, el uso de Internet sería una herramienta potencial de participación e inclusión de las personas con parálisis cerebral, sin dejar de lado los criterios de accesibilidad y usabilidad, basado en un diseño universal y ajustes razonables de sus plataformas y páginas web (Chamorro y Silvero, 2014; Ferreira et al., 2012; Johansson et al., 2021; Newman et al., 2017).

Es importante acentuar que la socialización, desde una perspectiva de las ciencias sociales y humanidades, es un concepto flexible y dinámico, que puede entenderse como aquella capacidad del ser humano para construir relaciones sociales y poder asociarse, interactuar y satisfacer sus necesidades e intereses con otros sujetos e instituciones (Grusec y Hastings, 2015). De igual modo, dicho término puede ser concebido como uno de los canales de comunicación beneficioso para adquirir informaciones, conocimientos y emociones de acuerdo con las etapas y ciclos que va experimentando el individuo en el transcurso de su vida (Romeu, 2019; Simkin y Becerra, 2013).

Nuestra intención en este trabajo no es caer en conceptualizaciones, teorías y/o modelos epistemológicos que sean deterministas o estáticos sobre la socialización para adultos con parálisis cerebral en entornos virtuales o a distancia, sino más bien procurar mostrar evidencias sobre la conexión de Internet en sus vidas. Por lo tanto, el objetivo de este estudio es identificar las percepciones sobre el uso de Internet y su influencia en las experiencias de vida de adultos con parálisis cerebral. En otros términos, reflexionar sobre su utilidad como un insumo que promueve otras formas de pensar, sentir y actuar para las personas con discapacidad física.

\section{Metodología}

Este trabajo se enmarca en una investigación cualitativa de carácter descriptivo, en el cual se utilizaron entrevistas semi-estructuradas para su posterior análisis e interpretación (Ritchie et al., 2014). La utilidad de este tipo de entrevistas permite ceñirse no sólo a una guía de entrevista predefinida sino que permite reformular o agregar nuevos cuestionamientos en el desarrollo de ella. Es un estilo de entrevista flexible que posibilita sondear desde temas generales hasta temas específicos poco explorados (Kallio et al., 2016).

Estas entrevistas permitieron conocer y profundizar en las experiencias de vida de los participantes con parálisis cerebral sobre la forma de relacionarse con Internet. Dichas entrevistas se hicieron de modo presencial y virtual por videollamadas en Skype ${ }^{1}$, de acuerdo con la preferencia y disponibilidad del grupo de entrevistados y entrevistadas (Janghorban et al., 2014; lacono et al., 2016).

1. https://www.skype.com/. 
Fueron realizadas cinco entrevistas presenciales y siete entrevistas virtuales. Participaron doce adultos (cuatro mujeres y ocho hombres), específicamente, con parálisis cerebral del Estado de São Paulo, Brasil, y cuyas edades fluctuaron entre 21 y 35 años. La media de edad fue 29,41 años. Esta muestra se seleccionó de una manera no probabilística al ser una población con discapacidad invisibilizada (Huete, 2019). Es decir, no se busca realizar una cuantificación o medición probabilística de este grupo, sino comprender sus percepciones y significados sobre fenómenos o procesos sociales (Escudero y Cortez, 2018; MartínezSalgado, 2012).

A lo largo de este texto se utilizará la letra P para indicar el número de participantes con parálisis cerebral y sus características específicas como edad y tipo de parálisis cerebral. Del grupo de mujeres participaron dos adultas con parálisis cerebral cuadriplejia espástica (P4 y P9), una con parálisis cerebral diplejia espástica $(\mathrm{P} 10)$ y una con parálisis cerebral hemiplejia espástica $(\mathrm{P} 11)$. Del grupo de hombres participaron dos adultos con parálisis cerebral cuadriplejia espástica (P2 y P3), cuatro adultos con parálisis cerebral diplejia espástica ( $\mathrm{P} 5, \mathrm{P} 6, \mathrm{P} 7$ y $\mathrm{P} 8)$, un adulto con parálisis cerebral discinética $(\mathrm{P} 1)$ y un adulto con parálisis cerebral hemiplejia espástica (P12).

Para la recolección de información de las y los participantes se cumplió con los protocolos de consentimiento informado. El proyecto ${ }^{2}$ fue sometido a Comité de Ética y aprobado conforme al Consejo Nacional de Salud del país brasileño. En base a los criterios éticos de investigación con seres humanos se mantiene el anonimato de las y los participantes que no revele su identidad.

Las transcripciones de las entrevistas fueron organizadas y sometidas a un análisis de contenido con la ayuda del programa Atlas.ti ${ }^{3}$ en su versión 8 . Fueron identificadas las siguientes cinco categorías temáticas: relaciones interpersonales, bienestar emocional, visibilidad social, participación política y barreras actitudinales.

Adicionalmente, se agregaron fragmentos de las citas de las y los entrevistados para ejemplificar y contrastar con la literatura científica existente, así como para proporcionar nuevas luces o cuestionamientos de la información que fue recolectada. Las citas fueron traducidas del portugués al español según corresponda. Se utilizaron las pautas de edición de Luiz Marcuschi (2004)4. La edición de los relatos procuró mantener la fidelidad semántica, sintaxis y morfología de las transcripciones originales.

\section{Resultados}

\subsection{Relaciones interpersonales}

Las personas con discapacidad física, entre ellas, con parálisis cerebral, afrontan no sólo dificultades para movilizarse o trasladarse, sino que su habilidad manual y su comunicación oral o verbal pueden estar afec-

\footnotetext{
2. Este proyecto contó con la financiación de la Asociación Universitaria Iberoamericana de Postgrado (AUIP) de España y la Universidade Estadual Paulista (UNESP) de Brasil en el marco de Apoyo a Estudiantes de Doctorado del Extranjero (PAEDEx) en los años 2015-2019.

3. https://atlasti.com/.

4. Por ejemplo, se usó el símbolo /.../ para señalar un recorte en la transcripción. Asimismo, se usó el símbolo ( ) para señalar alguna(s) palabra(s) o frase(s) que aclare(n) mejor el contenido. Finalmente, se usó el símbolo (( )) para comentarios adicionales del investigador.
} 
tadas o comprometidas. No obstante, Internet es una herramienta vital para ampliar su red de contactos. Como ejemplo, se indica lo siguiente:

"/.../ A través de las redes sociales yo conozco personas /.../ Yo amplié mis conocimientos y mi experiencia técnica a través de ella. Sabes de las conferencias, conversaciones con personas de otros lugares, no sólo de tu casa /.../ Por ejemplo, existe un padre (brasileño) que vive en Nueva York (y) tiene un hijo con parálisis cerebral, y a través (de) las redes sociales conversamos, intercambiamos ideas, también me ha pedido que grabe un video para su página web /.../ Pero está bien, porque es un tipo que no conozco /.../ Es brasileño y fue allí para hacer un tratamiento para su hijo. Él y su esposa tienen un sitio web llamado mãeespecial.com.br /.../ Doy conferencias sobre derecho y motivación. Muchas veces damos conferencias a 100, 300, 400 personas. Ellos ven /.../ y te agregan (en Facebook $)^{5}$. Personas que nunca he visto envían mensajes. Ellos dan retroalimentación de la conferencia del evento /.../ Tengo varios grupos de interés ((El participante me muestra en su computadora tradicional)). Tengo un grupo de investigación de la Maestría. Aquí la gente pone algunas cosas, aquí hay un grupo de parálisis cerebral que uso mucho, que tiene veinte mil personas /.../" (P1, 28 años, adulto con parálisis cerebral discinética).

“/.../ He conocido a muchas personas a través del computador, a través de Internet /.../ Me he comunicado con gente que está lejos, y también he conocido a otras personas /.../ Del extranjero, de todo tipo /.../ Utilizo más Facebook y WhatsApp 5 /.../ Tengo varios colegas con alguna discapacidad /.../ Los conocí en la propia Internet, a través de Orkut $^{6} / . . . /$ En una videollamada encontré a un tipo guapo, que me llamó la atención, pero no llegó a nada. Era sólo una amistad, nada más. Entonces, hubo un tiempo en que vino aquí. Nos conocimos en persona /.../ Tuve una (otra) experiencia con un chico, que vivía lejos. Funcionó durante unos 5 meses /.../ (Ya) conocí a unos amigos virtuales /.../ Conocí a un tipo sin discapacidad por una de esas aplicaciones de celular /.../" (P4, 28 años, adulta con parálisis cerebral cuadriplejia espástica).

"/.../ Confieso que antes de estas tecnologías me comunicaba menos /.../ Así que Facebook, WhatsApp, Orkut, y todo lo demás, me ayudaron aún más en mi comunicación, porque así podía hablar con las personas. Porque lo quiera o no, conversar es más fácil para mí por medio de un mensaje que hablar o escuchar /.../" (P12, 35 años, adulto con parálisis cerebral hemiplejia espástica).

Otro aspecto a destacar de los relatos de las y los entrevistados fue la utilidad de Internet que les permitió establecer relaciones sentimentales de personas con o sin discapacidad. Se detalla lo siguiente:

"(Con las redes sociales) conseguí incluso mi primera novia /.../ Era virtual y pasó después a lo real /.../ (Ella) era normal, $\sin$ (discapacidad) /.../ Ella vio mi foto y me percibió guapo. Tenía miedo. Nunca había conocido a nadie antes, así que tenía miedo. Entonces dije: "Veamos qué pasa". Vivía en un edificio en el centro de la ciudad /.../ Le di la dirección y ella vino a mi edificio. Allí, hablamos, y luego (comenzó) el noviazgo /.../ Fue a través de la primera red social que usé: el Orkut (que la conocî” (P2, 35 años, adulto con parálisis cerebral cuadriplejia espástica).

"Una de las buenas experiencias que he tenido en las redes sociales es que conocí a mi actual novio. Llevo dos años con él. Nos conocimos en un grupo (de Facebook) relacionado a la discapacidad física /.../ Cuando te unes a un grupo, automáticamente te muestra todos los miembros que ya participan en él. Entonces, lo añadí ((risas)). Después de unos minutos, él estaba en línea en Face (Facebook), y fui a hablar con él. Ahí tuvimos nuestra primera conversación, que comenzó a las 9 de la noche y terminó a las 5 de la mañana. Desde entonces, no hemos dejado de conversar. Hasta que llegó el Carnaval y quiso conocerme personalmente. No lo creía /.../ Y realmente vino, se

5. https://www.whatsapp.com/.

6. http://www.orkut.com/index.html. 
quedó aquí en un hotel y conoció a toda mi familia. Recuerdo que antes de irse me preguntó si quería salir con él. Así que le dije que le daría la respuesta sólo cuando se fuera ((risas)). Cuando vino a pasar los cuatro días de Carnaval ((risas)), nos fuimos, fuimos al centro comercial. Le mostré todo lo que mi ciudad ofrece. Luego nos quedamos en el restaurante. Cuando se fue, me preguntó otra vez si íbamos a seguir juntos o si sólo seríamos amigos. Pensé, pero no dije: "No tengo nada que perder, veré qué pasa" ((risas)) /.../ Vamos a cumplir dos años ahora en enero y el año que viene tenemos intención de casarnos /.../" (P10, 33 años, adulta con parálisis cerebral diplejia espástica).

\subsection{Bienestar emocional}

Aparte de la categoría descrita previamente, se muestra que la utilización de Internet proporcionó a este grupo de adultos con parálisis cerebral un bienestar emocional que tuvo un efecto positivo para su autoestima. Como ejemplo, se señala lo siguiente:

"/.../ Yo tengo parientes en X (ciudad de Brasil) y en otros lugares /.../ Entonces, yo uso las redes sociales para reanudar la comunicación, en lugar de sólo llamar, lo cual es costoso /.../ Eso facilita en este caso /.../ Nos mantenemos conectados con el mundo. Puedes conocer al pariente que está lejos, puedes conversar. Son muchos (aspectos) positivos /.../ (En el trabajo) la experiencia fue demasiado buena, porque allí me desarrollé aún más. Allí me hice amigo de colegas con discapacidad, y también de aquellos que no tienen ningún tipo de discapacidad. Conversaba. Ellos me ayudaban en todo. Hasta hoy tengo contacto con ellos en Face (Facebook), Whats (WhatsApp), Instagram Tenemos un grupo en Whats (WhatsApp). Cuando vamos a realizar una confraternización, hacemos un grupo. El grupo de hombres se llama el "Grupo X" ((risas)). Luego cambiaron otro nombre: "El grupo de las Y". Ellos siempre se ponen en contacto conmigo /.../ (La red social ha ampliado mis contactos), porque solía hablar sólo con la gente de mi casa, pero luego Facebook trajo más posibilidades" (P6, 31 años, adulto con parálisis cerebral diplejia espástica).

Dicha estabilidad emocional para estas personas con discapacidad física sería reforzada por el uso de Internet, ya sea para afrontar momentos de depresión o etapas de desánimo. Se explica lo siguiente:

“/.../ Antes, cuando era más joven tenía un blog /.../ Era un blog donde publicaba mensajes de optimismo /.../ Eran frases de autoayuda. Luego, terminé creando un blog donde podía publicar cosas para que otras personas las vieran /.../Eran frases que me gustaban /.../ Era un blog con frases de autoayuda dirigido a personas con discapacidad /.../ Cuando tenía 19 años sufría de depresión. Después, conocí este grupo de chat (de $\mathrm{UOL}^{8}$ ). Cuando entré en la sala de chat, conocí a un tipo que estaba en la TV /.../ Incluso antes del chat no salía con gente. No frecuentaba ninguna asociación. No hacía nada de eso. Sólo iba a la clínica (para) hacer fisioterapia, y allí vi a personas con discapacidad, pero no tuve mucho contacto con ellos /.../ Todo el contacto que tuve fue en la red social /.../ Creo que cuando haces amigos pasas un poco de tu experiencia de vida a otra persona. Si sabes absorber los puntos positivos, puedes ver la vida de una manera diferente. Incluso si es una experiencia mínima, si puedes absorberla puedes ayudar a otras personas /.../" (P10, 33 años, adulta con parálisis cerebral diplejia espástica).

7. https://www.instagram.com/

8. https://batepapo.uol.com.br/. 


\subsection{Visibilidad social}

Otro aspecto de importancia con el uso de Internet para las personas con parálisis cerebral entrevistadas es la posibilidad de visibilizar su condición a través de este medio. Como ejemplo, se afirma lo siguiente:

"Hice un video en mi sitio web (en Facebook) y cualquiera que quiera verlo puede unirse a mi clase /.../ Así es como surgió la idea. Desde los 20 años doy conferencias. Desde el segundo, tercer año de graduación. Tengo una buena facilidad con la oratoria /.../ Entonces la idea que surgió este año (2017) fue mía. Lo hago de manera trivial /.../ Así que, algunas veces, señalo algunas cosas, doy charlas motivacionales, política /.../ Algo no tan teórico /.../ Hay personas que dicen: "Estoy disfrutando de tus artes" /.../ Hay un colega mío (con una discapacidad) que, mientras veía uno de mis videos, se sintió motivado a dejar de lado la tristeza y seguir adelante /.../" (P1, 28 años, adulto con parálisis cerebral discinética).

“/.../Tuve una colega que también usa silla de ruedas /.../ Tiene un canal en Youtube 9 . Su canal es Y /.../ No habla bien porque tiene parálisis cerebral /.../ Es famosa ((risas)), debe tener 18 años /.../ Es ella la que publica (los videos) /.../ Somos amigas /.../ Es amistad de verdad (en la red social) /.../" (P9, 34 años, adulta con parálisis cerebral cuadriplejia espástica).

De este modo, se propone que el uso de Internet para las personas con parálisis cerebral puede favorecer a combatir los prejuicios o tabúes sobre su condición. Se enuncia lo siguiente:

"(Internet) ayudó mucho. Descubrí un conocimiento que no tenía sobre la computadora. Me ayudó a crear mi (canal) de Youtube, a poner mi cara, mis videos. La mayoría de las personas saben que uso silla de ruedas /.../ Tengo una página en Face (Facebook), donde publico videos, que es lo mismo que el canal (de Youtube) /.../ Así, la gente ve que una persona con discapacidad hace todo: enamora, besa. Hace todo lo que quiere, incluso cosas que no debería. La gente piensa que una persona con discapacidad no hace nada, no tiene relaciones sexuales, (que) es un tonto /.../" (P2, 35 años, adulto con parálisis cerebral cuadriplejia espástica).

"Veo que se quebró un poco el tabú de la discapacidad (en las redes sociales). Tienes grupos en Facebook que se llaman "personas con discapacidad". Conocí a X (un amigo que usa silla de ruedas) a través de las redes sociales. Creo que los grupos de Facebook y el hashtag (etiqueta) terminaron reuniendo a personas con discapacidad, con intereses similares /.../ Los amigos con discapacidad que yo tuve los conocía en la escuela o haciendo algún tratamiento. Creo que las redes sociales te permiten conocer no sólo a personas con discapacidad, sino también a personas con intereses en determinados temas sobre discapacidad /.../ En realidad, mi blog comenzó porque cuando busqué en Google ${ }^{10}$ a personas con discapacidad, el tema no era tratado de una manera /.../ feliz. Y esa es la propuesta de mi blog, tanto que el símbolo es muy colorido. El blog fue (para) tratar los problemas de accesibilidad e inclusión de una manera alegre. En el caso de Youtube, estoy recientemente desarrollando un proyecto en mi canal. Estoy desarrollando un proyecto de actividad física /... / Es un debate en línea sobre la actividad física adaptada para las personas con discapacidad" (P11, 27 años, adulta con parálisis cerebral hemiplejia espástica).

9. https://www.youtube.com/.

10. https://www.google.com/. 


\subsection{Participación política}

Internet para el grupo de discapacidad física entrevistado sería una herramienta que provee un modo eficiente para compartir información, pero, sobre todo, un medio eficaz para movilizar sus propias elecciones o toma de decisiones y viabilizar, en consecuencia, su autonomía y vida independiente. Como ejemplo, se subraya lo siguiente:

"/.../ Cuando llega el carro (de Uber $\left.{ }^{11}\right)$, los conductores me dicen: "Pide otro (Uber), porque la silla de ruedas no cabe en el carro". Por la aplicación, tienes la posibilidad de quejarte con la empresa. Entonces, escribo que el conductor no quería llevarme y que canceló el servicio porque no quería llevar la silla de ruedas. Cada vez que esto sucede me quejo en el aplicativo. La misma empresa me devuelve el valor y gano un servicio adicional /.../" (P10, 33 años, adulta con parálisis cerebral diplejia espástica).

Particularmente, el uso de Internet se desempeñaría como un espacio para hacer respetar sus derechos legales que son, en ocasiones, vulnerados. Es decir, Internet les permite una acción política y empoderamiento para realizar denuncias públicas que, en otros momentos, circunstancias u oportunidades de sus vidas, les hubiese sido difíciles de mostrar o llevar a cabo. Se muestra lo siguiente:

"/.../ Por ejemplo, tuve un caso reciente. Eso me llamó mucho la atención. Tengo una demanda contra X (aerolínea), porque tengo un descuento por tener una discapacidad, pero no me lo dieron, no reconocieron mi discapacidad. Entonces, ¿qué pasó? Recibí la respuesta oficial de la compañía, escribí un texto y lo publiqué en mi línea de tiempo (de Facebook). Tenía más de 330 "me gusta" y sesenta y algo compartimientos. Vi a la Asociación X (Asociación de Derechos Humanos) compartiendo mi publicación. Algunas madres comentaron que lo mismo había sucedido con sus hijas con discapacidad. Gente de la asociación que nunca conocí. Pero tú generas esa cadena, incluso debido a los propios algoritmos de Facebook /.../ Recibí un correo electrónico de una madre en la ciudad X (ciudad de otro estado), preguntando si conocía a algún abogado que trabajara allí, ¿entiendes? /.../ Es bastante genial /.../ Veo la red social como un espacio para el debate" ( $\mathrm{P} 1,28$ años, adulto con parálisis cerebral discinética).

"Existe una aplicación llamada Guia de rodas'12 (Guía de ruedas). En ella, pones si un restaurante, bar, hotel tiene accesibilidad. Es una aplicación gratuita /.../ Es sólo de Brasil /.../ Ya he publicado algunas cosas en ella. Por ejemplo, si voy a un bar que sólo tiene una escalera y no puedo subir las escaleras, no puedo ir allá. Si no hay un baño accesible, si los empleados ayudan a cortar la comida o no ayudan" (P11, 27 años, adulta con parálisis cerebral hemiplejia espástica).

\subsection{Barreras actitudinales}

Específicamente, uno de los entrevistados con parálisis cerebral reportó una de las tendencias actuales de bullying por Internet: el cyberbullying. Como ejemplo, se expone lo siguiente:

“/.../ La dificultad (que tengo) es la dicción. Mi voz, mi forma de hablar (en mis videos de Youtube), lo encuentro prejuicioso. Por ejemplo, la persona no lo hace, pero critica a quien lo hace. Creo que es un poco complicado. Para que la persona lo critique ella tiene que hacerlo mejor que yo. Eso no sucede, prefieren destruirte, en vez de decir: "Hazlo así, habla con más calma" /.../ (En los comentarios) dicen: "Tu vida es mala, tu vida es una m /.../, eres un mongol".

11. https://www.uber.com/.

12. https://guiaderodas.com/. 
Ese tipo de bullying /.../ Yo digo que no estoy haciendo un canal de personas con silla de ruedas. Si hubiera hecho ese tipo de canal, hablaría, no me avergüenzo /.../ Quien quiere lo ve. Si nadie quiere, paciencia, nadie es perfecto /.../ Entonces (dicen): "Él es una persona con silla de ruedas" y se compadecen. Siguen preguntando y haciendo bullying /.../" (P2, 35 años, adulto con parálisis cerebral cuadriplejia espástica).

Por otra parte, una de las entrevistadas con parálisis cerebral puntualizó sobre el acoso de personas sin discapacidad que buscan intimidad o relaciones sexuales a través de las redes sociales. Se manifiesta lo siguiente:

"/.../ Son hombres o mujeres sin limitaciones físicas, que dicen sentirse atraídos por personas con discapacidad física. Pero es una atracción sexual. En este caso, la limitación de una persona con discapacidad atrae a estas personas /.../ En la sala de chat de UOL que indiqué, hay muchas personas así /.../ Son personas sin limitación física que se sienten atraídas por las personas con discapacidad /.../ (En Facebook) también hay /.../ Es que, de hecho, van donde están las personas con discapacidad, que son su foco /.../” (P10, 33 años, adulta con parálisis cerebral diplejia espástica).

A dichas barreras actitudinales, se suma la creencia sobre aquello que puede hacer o no la persona con parálisis cerebral a través de Internet. Como ejemplo, se demuestra lo siguiente:

"Hay personas que piensan que no escribo en Facebook. Creen que es mi madre y me dicen: “¿Quién escribe por ti?” Digo: “yo” ((risas)), y ellos preguntan: “¿Dónde está tu mamá?”. Yo respondo: “Está trabajando” /.../ Yo misma he creado mi Facebook, mi contraseña /.../ Personas como amigos, parientes e incluso el profesor de X (institución de educación especial y/o rehabilitación) piensan que no escribo ((risas)). Escribo palabra por palabra, sólo que me lleva un tiempo, porque escribo con una sola mano /.../ Como no puedo escribir allá (institución de educación especial y/o rehabilitación) la gente piensa que no escribo (en la tablet) /.../" (P9, 34 años, adulta con parálisis cerebral cuadriplejia espástica).

"/.../ Diría que la mayoría de las personas piensan que tengo algún tipo de discapacidad intelectual. He pasado la mayor parte de mi vida luchando contra ello. Cuando la persona me conoce, la idea de que tengo una discapacidad intelectual termina /.../ Cuando necesitaba hablar con alguien que no me conocía, normalmente pensaba que tenía algún problema mental. Ahora, cuando estoy con amigos y parientes, todos piensan que soy normal. Hablaban y se quedaban conmigo normalmente /.../ Así que, de hecho, esto siempre sucede, en cualquier lugar, ya sea en la escuela, en el cine, en el mercado, en la universidad, en las redes sociales /.../ Queriendo o no, la discapacidad visual y auditiva termina siendo más común que la parálisis cerebral. La persona con parálisis cerebral termina atrayendo mucho más la atención /.../" (P12, 35 años, adulto con parálisis cerebral hemiplejia espástica).

\section{Discusión}

Respecto al primer eje temático de "relaciones interpersonales" se sugiere que, cotidianamente, los adultos con parálisis cerebral enfrentaron limitaciones para su inclusión social de modo presencial debido al estigma de su misma condición física y probable aislamiento (Suriá, 2012, 2017; Villalobos et al., 2016). Sin embargo, Internet a este tipo de personas les facilita un medio de comunicación relevante para mantener contacto 
con sus pares y expandir sus relaciones sociales e interacciones con conocidos y desconocidos, lo cual fomentaría su participación e inclusión social (Caron y Light, 2015; Newman et al., 2017; Rasid y Nonis, 2015).

Las personas con esta condición de discapacidad usualmente son excluidas o rechazadas para establecer una vida amorosa y/o sexual en virtud de ciertos estereotipos negativos impuestos por la sociedad (Huskin et al., 2017; Wiegerink et al., 2006). Esta óptica se entrecruza con una desinformación que denota una discriminación para este grupo de personas, el cual no cree que pueda ejercer dichos derechos. Además, pone al descubierto una ausencia de programas de educación sexual que no motivan necesariamente esta apertura y disfrute de su libertad sexual de manera plena y saludable (Ponsa et al., 2018).

Con relación al segundo eje temático de "bienestar emocional" es relevante mencionar que las investigaciones para adultos con discapacidad física, entre ellas, con parálisis cerebral midan o verifiquen dentro de sus indicadores las ventajas o desventajas del uso de Internet u otras tecnologías; y que lo relacionen con su calidad de vida (Alves-Nogueira et al., 2020; Maestro-González et al., 2018; Mendes et al., 2018).

Es importante destacar que la literatura científica indica que el grupo de adultos con parálisis cerebral tiene un potencial riesgo de desarrollar índices de ansiedad y depresión. No obstante, sus causas necesitan mayores estudios sociales, que permitan a futuro considerar si Internet implica o no un rol beneficioso para evitar dichas emociones y sentimientos (Smith et al., 2019).

Respecto al tercer eje temático de "visibilidad social" se puede destacar que Internet para las personas con parálisis cerebral se convierte en un "espacio liberador" para la muestra y/o expresión de su discapacidad física (Suriá, 2012). Es decir, es un recurso que fortalecería su presencia mediática para ser conocidos en base a sus proyectos personales, que utilizan mensajes textuales y/o audiovisuales. Todo ello es difundido en los entornos virtuales, que son complementarios a los medios de comunicación tradicionales como la televisión y la radio (Ferreira et al., 2012; Shah et al., 2020).

Históricamente, la discapacidad se ha presentado, dentro y fuera de los medios de comunicación, a adjetivaciones que son encasilladas o enmarcadas a sus características cognitivas, físicas y/o sensoriales; y no en sus potencialidades (Worrell, 2018). Este tipo de cualidades están dadas por cuestiones culturales, por falta de una política pública y social clara sobre educación inclusiva y el respeto a la diversidad, así como por el énfasis de un modelo médico-rehabilitador que prioriza el foco en las necesidades funcionales y físicas de las personas (Jiménez, 2017; Sentenac et al., 2013; Solís y Real, 2019).

Para ello, se deben promover propuestas de sensibilización, diálogo e interacción de las personas con o sin discapacidad, entre ellas, con parálisis cerebral, en diferentes contextos presenciales y virtuales. En otros términos, que se problematice la concientización de una igualdad y equidad para todas y todos independientemente de su discapacidad (García et al., 2011; Leutar y Raič, 2008; Tonugble et al., 2021).

Con relación al cuarto eje temático de "participación política" las personas con parálisis cerebral enfrentan no sólo obstáculos en los entornos por los cuales transitan debido a la falta de accesibilidad física, sino también con determinados productos y/o servicios que no están adaptados a sus particularidades (Braccialli et al., 2016; Schur y Adya, 2013). De esta forma, se debe procurar seguir los modelos o criterios de diseño universal para satisfacer las necesidades de las personas con discapacidad (Chamorro y Silvero, 2014; Ferreira et al., 2012; González-Alonso y García-Alonso, 2014; Johansson et al., 2021; Newman et al., 2017). En este punto, Internet se convierte en un instrumento político para manifestar sus quejas y velar así por el respeto de sus derechos. 
Finalmente, respecto al quinto eje temático de "barreras actitudinales" se evidencia la exclusión que se puede dar en Internet para este grupo de personas. Por ejemplo, las personas con discapacidad física, por determinados rasgos de su cuerpo o formas de actuar, son consideradas injustamente como "no normales o no comunes", convirtiéndose en un motivo o señal de discriminación para aquellas o aquellos que no cuentan con dicha condición (Inahara, 2009; Tonugble et al., 2021). Asimismo, se puede encontrar algún tipo de acoso virtual. Por consiguiente, se sugiere una discriminación no sólo por pertenecer o ser de determinado género, sino que también su discapacidad conllevaría otras vulnerabilidades (Mueller-Johnson et al., 2014).

Complementariamente, se señalaron las percepciones de personas sin discapacidad sobre lo que podría hacer o no con Internet la persona con parálisis cerebral. Esto reafirma los prejuicios vigentes sobre la discapacidad física que no valoriza sus habilidades y competencias y/o que las personas sin discapacidad las desconoce o que creen que no son capaces de realizarlas (González-Alonso y Matía, 2018).

\section{Conclusiones y recomendaciones}

Los estudios sobre adultos con parálisis cerebral y el uso de Internet presentan ausencias en la literatura internacional que merecen atención de la comunidad científica de las diversas áreas del conocimiento. La socialización para este tipo de personas está tomando otras formas o estilos que no son únicamente "cara a cara" o de modo presencial, sino que surgen y evolucionan a través de Internet. Su utilidad sobrepasa el espacio físico y las fronteras geográficas, adoptando nuevos sentidos y significados para la vida de las personas con discapacidad física. Es oportuno indicar que Internet sería una herramienta para reforzar y generar vínculos sociales, así como relaciones de enamoramiento e intimidad complementarias a otros contextos.

Asimismo, se sugiere que Internet significa un recurso esencial que puede ser aliado para la mejora de la salud mental y bienestar psicológico de adultos con parálisis cerebral. Este aspecto sería un potencial indicador en el cual futuras investigaciones puedan incluir en sus instrumentos de medición esta herramienta tecnológica como medio de desarrollo para su calidad de vida.

Por lo señalado, Internet permite posibilidades de visibilidad social, inclusión y participación política a este grupo poblacional. Es valioso resaltar el uso de las redes sociales por los adultos con parálisis cerebral para manifestar alguna denuncia pública o crítica a situaciones que transgreden sus derechos como personas con discapacidad. Esto invitaría a nuevos estudios sobre la autorepresentación y representación de las personas con esta condición en las redes sociales. Y, en otros casos, puede revelar tipos de bullying y posibles daños o perjuicios que puedan violentar, denigrar o difamar a estas personas en el mundo digital.

Se recomienda indagar diversas maneras de socialización presencial y/o virtual que puedan vincularse con ambientes o lugares como el educativo, laboral, recreativo y/o de terapia y rehabilitación. Además, se sugiere ampliar este estudio con otras muestras significativas para recabar otras experiencias de vida que muestren las realidades de otros países. Todo ello permitiría fortalecer e incrementar los estudios cualitativos y cuantitativos sobre discapacidad y su relación con el uso de Internet. 


\section{Referencias bibliográficas}

Alves-Nogueira, A. et al. (2020). A systematic review on quality of life assessment in adults with cerebral palsy: Challenging issues and a call for research. Research in Developmental Disabilities, 96, pp. 1-20. https://doi. org/10.1016/j.ridd.2019.103514.

Braccialli, L. et al. (2016). Acesso ao computador por crianças e jovens com paralisia cerebral. Educação, Formação \& Tecnologias, 9(1), pp. 72-84.

Cabral, U. (26 de agosto de 2021). Um em cada quatro idosos tinha algum tipo de deficiência em 2019. IBGE. https://agenciadenoticias.ibge.gov.br/agencia-noticias/2012-agencia-de-noticias/noticias/31447-um-em-cada-quatro-idosos-tinha-algum-tipo-de-deficiencia-em-2019.

Caron, J. y Light, J. (2015). Social media has opened a world of 'Open communication': Experiences of adults with cerebral palsy who use augmentative and alternative communication and social media. Augmentative and Alternative Communication, 32(1), pp. 1-16. https://doi.org/10.3109/07434618.2015.1052887.

Chamorro, M. y Silvero, J. (2014). Enfoque de la Convención internacional sobre los derechos de las personas con discapacidad: las tecnologías de la información y comunicación como elemento de inclusión social. Rev. Int. Investig. Cienc. Soc., 10(2), pp. 239-262. https://dialnet.unirioja.es/servlet/articulo?codigo=4934380.

Escudero, C. y Cortez, L. (Coords.) (2018). Técnicas y métodos cualitativos para la investigación científica. Universidad Técnica de Machala.

Ferreira, A. et al. (2012). Accessibility for people with cerebral palsy: The use of blogs as an agent of social inclusion. Procedia Computer Science, 14, pp. 245-253. https://doi.org/10.1016/j.procs.2012.10.028.

García, T. et al. (2011). The use of computers and augmentative and alternative communication devices by children and young with cerebral palsy. Assistive Technology: The Official Journal of RESNA, 23(3), pp. 135-149. https:// doi.org/10.1080/10400435.2011.588988.

González-Alonso, M. y García-Alonso, M. (2014). La accesibilidad como la necesidad más percibida por las personas con parálisis cerebral en proceso de envejecimiento. International Journal of Developmental and Educational Psychology, 2(1), pp. 311-318.

González-Alonso, M. y Matía, A. (2018). Características de los usuarios de la Asociación de Parálisis Cerebral. Medicina de Familia. SEMERGEN, 44(8), pp. 557-561. https://doi.org/10.1016/j.semerg.2018.07.003.

Grusec, J. y Hastings, P. (Eds.) (2015). Handbook of Socialization. Theory and Research. The Guilford Press.

Heidrich, R. et al. (2014). Neuro-Navegática: software desenvolvido para interação com brain-computer interface para auxiliar o processo de inclusão escolar de pessoas com paralisia cerebral. Educação Temática Digital, 16(2), pp. 287-306.

Heidrich, R. y Bassani, P. (2012). Inclusive design-assistive technology for people with cerebral palsy. Work, 41, pp. 4762-4766. https://doi.org/10.3233/WOR-2012-0028-4762.

Huete, A. (2019). Autonomía e inclusión de las personas con discapacidad en el ámbito de protección social. Banco Interamericano de Desarrollo. 
Huskin, P. et al. (2017). Attitudes of undergraduate students toward persons with disabilities: Exploring effects of contact experience on social distance across ten disability types. Rehabilitation Counseling Bulletin, 62(1), pp. 53-63. https://doi.org/10.1177/0034355217727600.

lacono, V. et al. (2016). Skype as a tool for qualitative research interviews. Sociological Research Online, 21(2), pp. 103-117. https://doi.org/10.5153/sro.3952.

Inahara, M. (2009). This body which is not one: The body, femininity and disability. Body \& Society, 15(1), pp. 4762. https://doi.org/10.1177/1357034X08100146.

Janghorban, R. et al. (2014). Skype interviewing: The new generation of online synchronous interview in qualitative research. International Journal of Qualitative Studies on Health and Well-Being, 9(1), pp. 1-4. https://doi. org/10.3402/qhw.v9.24152.

Jiménez, A. (2017). Efectos de las terapias ecuestres en personas con parálisis cerebral. Revista Española de Discapacidad, 5(2), pp. 171-184.

Johansson, S. et al. (2021). Disability digital divide: the use of the Internet, smartphones, computers and tablets among people with disabilities in Sweden. Universal Access in the Information Society, 20, pp. 105-120. https://doi.org/10.1007/s10209-020-00714-x.

Kallio, H. et al. (2016). Systematic methodological review: developing a framework for a qualitative semi-structured interview guide. Journal of Advanced Nursing, 72(12), pp. 2949-3217. https://doi.org/10.1111/jan.13031.

Leutar, Z. y Raič, N. (2008). The influence of some socio-demographic characteristics of young people on their attitudes towards people with physical disabilities. SEER: Journal for Labour and Social Affairs in Eastern Europe, 11(4), pp. 517-538. https://doi.org/10.5771/1435-2869-2008-4-517.

Maestro-González, A. et al. (2018). Quality of life as assessed by adults with cerebral palsy. PloS ONE, 13(2), pp. 1-12. https://doi.org/10.1371/journal.pone.0191960.

Marcuschi, L. (2004). Da fala para a escrita: atividades de retextualização. Cortez Editora.

Martínez-Salgado, C. (2012). El muestreo en investigación cualitativa. Principios básicos y algunas controversias. Ciência \& Saúde Coletiva, 17(3), pp. 613-619. https://doi.org/10.1590/S1413-81232012000300006.

Mendes, A. et al. (2018). Qualidade de vida em pacientes adultos com paralisia cerebral. Acta Fisiátrica, 25(2), pp. 49-53. https://doi.org/10.11606/issn.2317-0190.v25i2a162559.

Mueller-Johnson, K. et al. (2014). Sexual victimization of youth with a physical disability: an examination of prevalence rates, and risk and protective factors. Journal of Interpersonal Violence, 29(17), pp. 3180-3206. https:// doi.org/10.1177/0886260514534529.

Newman, L. et al. (2017). Applying a critical approach to investigate barriers to digital inclusion and online social networking among young people with disabilities. Information Systems Journal, 27(5), pp. 559-588. https://doi. org/10.1111/isj.12106.

Oskoui, M. et al. (2013). An update on the prevalence of cerebral palsy: A systematic review and meta-analysis. Developmental Medicine \& Child Neurology. 55(6), pp. 509-519. https://doi.org/10.1111/dmcn.12080.

Panteliadis, C. (Ed.) (2018). Cerebral palsy. A Multidisciplinary Approach. Springer Nature.

Peixoto, M. et al. (2020). Características epidemiológicas da paralisia cerebral em crianças e adolescentes em uma capital do nordeste brasileiro. Fisioterapia e Pesquisa, 27(4), pp. 405-412. https://doi.org/10.1590/18092950/20012527042020. 
Ponsa, M. et al. (2018). Salud sexual de las personas con discapacidad física: educación sexual. International Journal of Developmental and Educational Psychology, 3(1), pp. 41-51. https://doi.org/10.17060/ijodaep.2018. n1.v3.1210.

Rasid, N. y Nonis, K. (2015). Exploring communication technology behaviour of adolescents with cerebral palsy in Singapore. International Journal of Special Education, 30(3), pp. 17-38.

Ritchie, J. et al. (Eds.) (2014). Qualitative research practice: A guide for social science students and researchers. Sage Publications Ltd.

Rodrigo, C. y Tabuenca, B. (2020). Ecologías de aprendizaje en estudiantes online con discapacidades. Comunicar, 62, pp. 53-65. https://doi.org/10.3916/C62-2020-05.

Romeu, V. (2019). Sociabilidad y sensibilidad en Simmel. Reflexiones desde la fenomenología de la comunicación. Estudios Sociológicos, 37(110), pp. 369-396. https://doi.org/10.24201/es.2019v37n110.1693.

Santos, T. et al. (2020). Eu esqueço que sou deficiente: interações e sociabilidade de adolescentes com deficiência física que praticam esportes. Cad. Saúde Pública, 36(2), pp. 1-12. https://dx.doi.org/10.1590/0102$311 \times 00014219$.

Schur, L. y Adya, M. (2013). Sidelined or mainstreamed? Political participation and attitudes of people with disabilities in the United States. Social Science Quarterly, 94(3), pp. 811-839. https://doi.org/10.1111/j.15406237.2012.00885.x.

Sentenac, M. et al. (2013). Determinants of inclusive education of 8-12 year-old children with cerebral palsy in 9 European regions. Research in Developmental Disabilities, 34(1), pp. 588-595. https://doi.org/10.1016/j. ridd.2012.09.019.

Shah, S. et al. (2020). Using Facebook to tell stories of premature ageing and sexual and reproductive healthcare across the life course for women with cerebral palsy in the UK and USA. BMJ Open, 10(2), pp. 1-7. http:// dx.doi.org/10.1136/bmjopen-2019-032172.

Simkin, H. y Becerra, G. (2013). El proceso de socialización. Apuntes para su exploración en el campo psicosocial. Ciencia, Docencia y Tecnología, 24(47), pp. 119-142.

Smith, K. et al. (2019). Risk of depression and anxiety in adults with cerebral palsy. JAMA Neurology, 76(3), pp. 294-300. https://doi.org/10.1001/jamaneurol.2018.4147.

Solís, P. y Real, S. (2019). Estado actual de investigación en parálisis cerebral y envejecimiento: revisión sistemática. Revista Española de Discapacidad, 7(2), pp. 103-122.

Suriá, R. (2012). Redes sociales online y su utilización para mejorar las habilidades sociales en jóvenes con discapacidad. Escritos de Psicología, 5(3), pp. 16-23. http://dx.doi.org/10.5231/psy.writ.2012.1809.

Suriá, R. (2017). Redes virtuales y apoyo social percibido en usuarios con discapacidad: análisis según la tipología, grado y etapa en la que se adquiere la discapacidad. Escritos de Psicología, 10(1), pp. 31-40. http://dx.doi. org/10.5231/psy.writ.2017.21403.

Tonugble, C. et al. (2021). Experiences of adults living with cerebral palsy in Accra Ghana. Journal of Preventive and Rehabilitative Medicine, 3(1), pp. 60-72. https://doi.org/10.21617/jprm2021.321.

Villalobos, J. et al. (2016). Terapia de lenguaje oral y comunicación aumentativa y alternativa en pacientes con parálisis cerebral espástica. Rev. Mex. AMCAOF, 5(2), pp. 47-52. 
Wiegerink, D. et al. (2006). Social and sexual relationships of adolescents and young adults with cerebral palsy: A review. Clinical Rehabilitation, 20(12), pp. 1023-1031. https://doi.org/10.1177/0269215506071275.

Worrell, T. (2018). Disability in the Media: Examining stigma and identity. Lexington Book. 